\title{
Fingertip top display system based on Persistence of vision
}

\author{
Wen Jing, Liu Hao*, Yuan Lingjuan, Wang Zixuan, Ren Zhigang \\ Beijing Institute of Fashion, Beijing China \\ gxyliuh@bift.edu.cn
}

Keywords: POV; SCM; sensor; LED; MCU

Abstract: The LED display bar, such as a "fingertip top", is a "high-tech" toy made using visual retention effects. At rest, it's just a couple of LED light-emitting diodes (or LEDs), and as it rotates at a certain frequency, it changes (lights on or off) depending on where it is, eventually displaying an image or a string. This design makes an LED display bar. LED lights are arranged in a linear way, and the fan section formed when rotating can be used to distinguish Chinese characters or patterns such as "fierce my country", "me and my chasing dream", rotating speed, love, etc., and the display content can be switched with the button.

\section{Introduction}

This design takes STC's 8-bit microcontroller STC 15w204s as the control core, USES 5V battery power supply, and takes 12 diodes vertical arrangement as the display module. The basic principle is to use the visual retention effect of the human eye. LED light emitting tube serves as the display of each column of the picture, playing the role of scanning. When the fingertip top rotates, the mercury switch will turn on or off regularly with the swing, thus triggering the interrupt service program. The interrupt service program will call the display program to display the corresponding content. When the speed of shaking matches the speed of the program execution, what people see is a complete picture.

\section{Ease of Use}

All electronic components are assembled on 1 PCB. According to the graphic characters on the front of the circuit board inserted into the components, and then soldered on the back of the circuit board can be made successfully, the battery is also fixed on the circuit board.

Production steps:

1) Install the resistor first.

2) Install the crystal oscillator in the position marked "B" on the circuit board.

3) Install two 30pF ceramic capacitors at the position marked "C".

4) Soldering integrated circuit (IC) socket. After the correct insertion, use electric iron to weld the pin of IC socket firmly.

5) Next, install 12 light-emitting diodes. 
6) Install the jumper column on the lower left side of the IC socket.

7) Next, install the button switch.

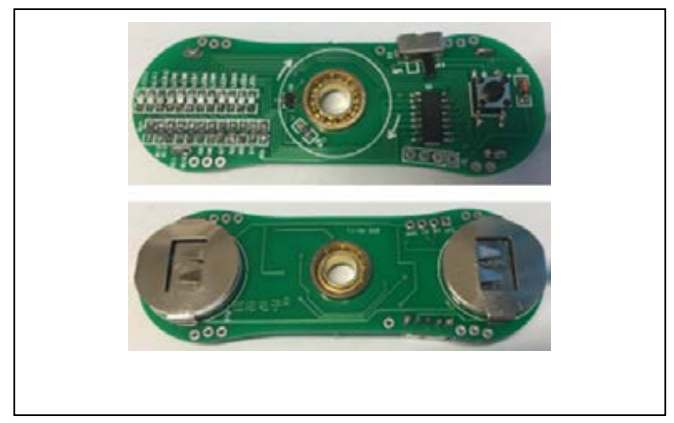

Figure 1 Circuit board

\section{Hardware System Design}

\subsection{System block diagram of the circuit}

Figure 1 shows the circuit diagram of the fingertip gyroscope. The main circuit is actually adding 12 LEDs and a mercury switch as a sensor to the MCU's minimum system.

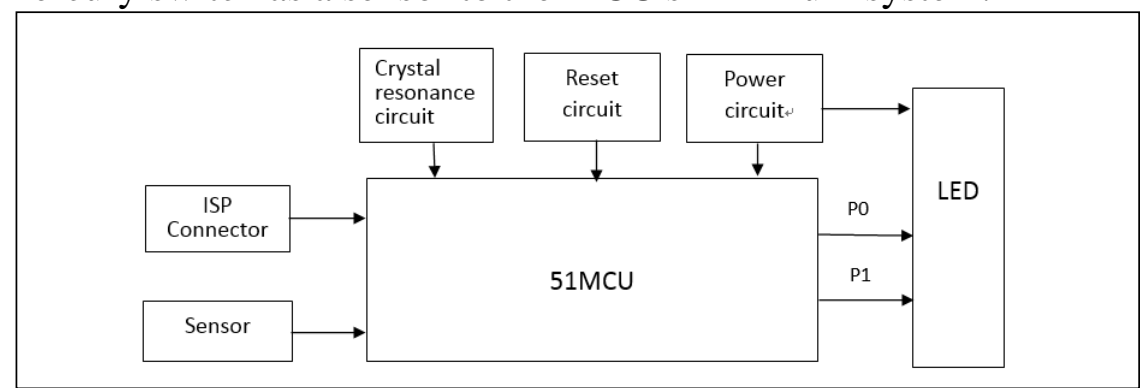

Figure 2 Diagram of fingertip gyroscope circuit

\subsection{Circuit schematic diagram}

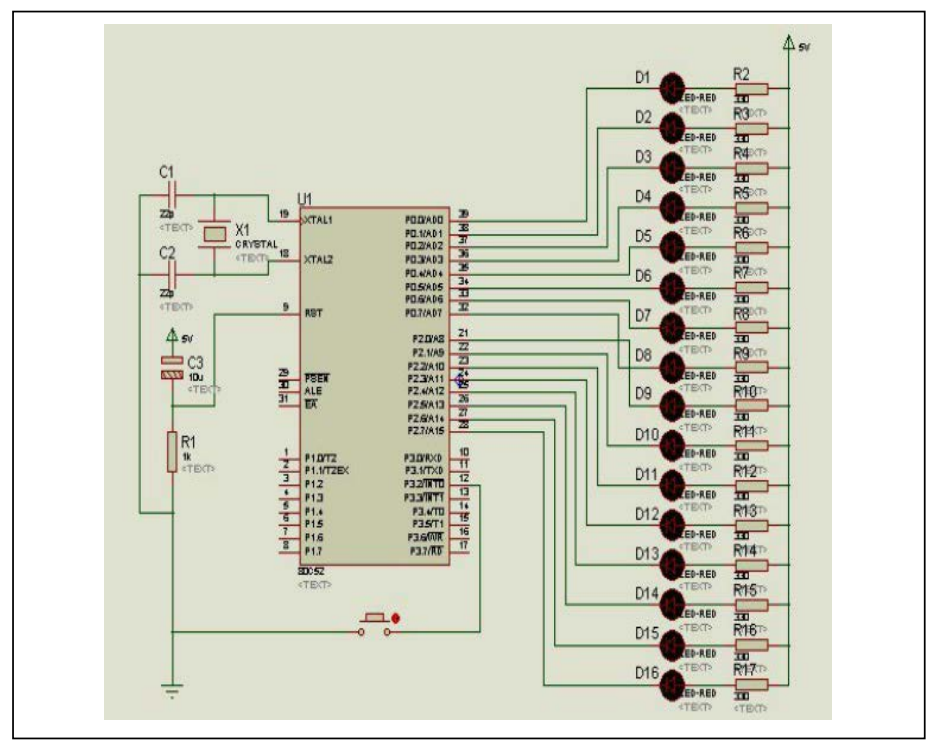

Figure 3 Diagram of fingertip gyroscope hardware connection 
As shown in figure 3, the fingertip gyro takes STC89C52 microcontroller as the control core and connects 16 highlighted light-emitting diodes through P0 and P2 port. The circuit diagram in figure 3 is mainly composed of reset circuit and crystal oscillator circuit.

\section{Software System Programming}

\subsection{Structure of software design}

The software consists of the function specification part, the delay subroutine part, the interrupt service routine part, the display subroutine part and the main function part.

(1) main function part: the initialization of the function is completed, and the display program segment is executed with the help of the while loop statement;

(2) declaration part: void display1(void);

(3) delay subroutine part:

void DelayUs (uint N)

$\{$ uint $\mathrm{x}$;

for $(\mathrm{x}=0 ; \mathrm{X}<=\mathrm{N} ; \mathrm{X}+)$;

There are 500ms between the first column display and the second column display.

(4) interrupt service program part: each shaking meter back mercury switch will generate a falling edge interrupt at both ends of the swing, and only extract one of them (it will be displayed from left to right);

(5) display subroutine part:

Void display1 (void)

\{ uchar I;

for (I - 0; I < 64; I++) \{P1 = dian [I * 2];

$\mathrm{P} 2=\operatorname{dian}[\mathrm{I} * 2+1]$;

DelayUs (500) :\}

\subsection{Program flow chart}

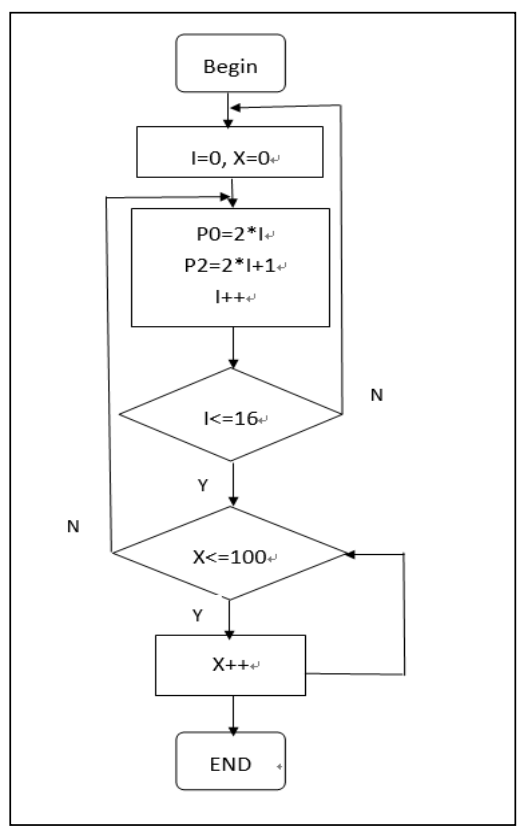

Figure 4 Flow chart 


\section{System Testing}

\subsection{Debugging process}

First, carefully check the circuit to see if there are any welding errors, and then plug in the battery. When welding hardware, the following errors often occur:

1) Connect the VCC end of the MCU directly to the anode of the $9 \mathrm{~V}$ battery, or even burn the MCU;

2) Mixed the pin order of 7805 so that $5 \mathrm{~V}$ voltage cannot be obtained;

3) Connect the positive electrode of the electrolytic capacitor to the ground end and the negative electrode to the high potential;

4) Forget to connect the MCU power supply and ground wire;

5) Connect the hexadecimal diode with the corresponding sequence of $\mathrm{P} 0$ and $\mathrm{P} 2$ port;

6) The mercury switch is not connected to the INT0 pin of the MCU.

\subsection{Test results}

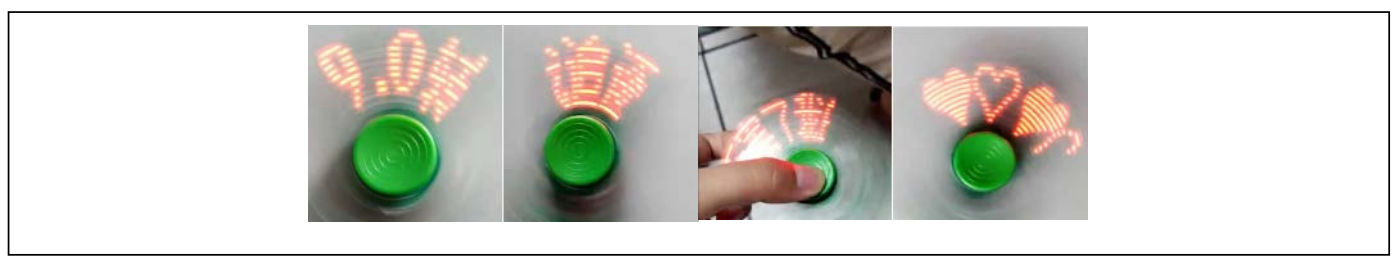

Figure 5 Text result

\section{Epilogue}

In this creative electronic design, the university's knowledge is fully demonstrated to be comprehensive, which is a challenging subject to cultivate students' independent thinking ability and flexible use of knowledge in various aspects. At the beginning, I felt that the subject given by the teacher was a little difficult, but life is a process of constantly overcoming difficulties. In the course design process, I found a lot of shortcomings, such as the Protel is not very sophisticated but only a little superficial, as well as not very good at mastering knowledge on books. However, I will correct my shortcomings in the future.

What impressed me the most was that the hardware and software were not well built, so the MCU could not be powered up and the program could not be written. Now I understand that theory alone is not enough, and doing according to the book is not enough, what we need more is practical ability and experience.

\section{Acknowledgment}

Our deepest gratitude goes first and foremost to Professor Liu, for his constant encouragement and guidance. He walked us through all the stages of the writing of this thesis.

Thanks for Open laboratory projects of Beijing Institute of Fashion Technology (NHFZ20180104/007).

Thanks for Postgraduate training base for talents outside school (120301990121/005).

\section{References}

[1] Wang Shan. Visual Basic programming course [M]. Mechanical industry press, January 2005. 
[2] Zhang Yikun. Principle and application of single-chip microcomputer [M]. Xidian University press, 1998, pp.78-92

[3] Du Huaichang, and Xiao Huaibao, and Huang Lingling, "Principle and application of CMOS integrated circuit", 2006, pp.157-160

[4] Chen Kailiang, and Zhu Shusheng, "Constant current source and its application circuit" Zhejiang science and technology press,1992, pp.67-68

[5] Han Zhijun " "MCU system design and application examples", China Machine Press,2010.3,pp.118-126

[6] He Limin," Application technology selection of single chip microcomputer”, BeiHang university Press,2006,p34

[7] Jiang Zhihong, "51MCU technology and application system development case selection",2008,pp.6-16,p146

[8] Macro crystal science and technology, P89V51RB2 serious, http://www.mcu-memory.com

[9] Ji Li, "Protel99SEschematic diagram and PCB design", Electronic Industry Press,2007,pp.116-119

[10] China electronic network, http://www.21ic.com 